\title{
Magnetic Field Alignment in the Beam-Beam Compensation Device
}

\author{
C. Crawford, A. Sery, V. Shiltsev, FNAL, Batavia, IL \\ A. Aleksandrov, B. Skarbo, B. Sukhina, BINP, Novosibirsk, Russia
}

\section{Abstract}

Guiding solenoidal magnetic field of the Tevatron beambeam compensation device has to be carefully aligned with respect to a straight trajectory of the antiproton beam. We present in this paper an optical method which allows to measure the direction of the magnetic field, results of magnetic measurements, and results of the field quality improvement with dipole correctors.

\section{INTRODUCTION}

Beam-beam interactions in the planned Tevatron upgrades Run II and TEV33 will cause betatron tune spread within bunch as well as bunch-to-bunch tune spread which will be high enough to enhance diffusion of particles due to high order resonances and to limit beam lifetime and luminosity.

Some of these beam-beam effects can be compensated by use of a counter-traveling electron beam ("electron lens") with appropriate charge distribution, which will apply additional linear or nonlinear defocusing for the antiproton beam, while it will pass through the electron beam [1]. Compensation of the beam-beam effects only for the antiprotons is sufficient since the proton bunch population is significantly higher than the antiproton bunch population.

Parameters of the electron beam for the beam-beam compensation are: beam current $J_{e}=2 \mathrm{~A}$, beam radius $a=$ $1 \mathrm{~mm}$, beam length $L_{e}=2 \mathrm{~m}$, energy $U_{e}=10 \mathrm{kV}$. The electron beam should be born in a weak magnetic field, and then, in order to provide sufficient "rigidness" of the electrons and also to increase the electron current density by means of the adiabatic magnetic compression of the beam, injected and transported in a strong longitudinal magnetic field. Considerations of electron beam distortions by the antiproton bunch and of a possible electron beam driven head tail instability in the antiproton beam suggested that the magnitude of the longitudinal magnetic field in the region where the antiprotons pass through the electron beam should be about 4-5 Tesla, while the field at the cathode of the electron gun should be about 0.1-0.2 Tesla [1].

\section{FIELD QUALITY REQUIREMENTS}

The electrons are highly magnetized in a guiding magnetic field of the beam-beam compensation device and thus follow the direction of the field, while the trajectory of the antiprotons, due to their high energy, is almost straight in the beam-beam compensation device. The straightness of magnetic field is therefore essential for the beam-beam compensation to work properly. Deviation of the electron trajectory from the straight line should be $\Delta x_{e} \lesssim 0.1 a$ that is about $0.1 \mathrm{~mm}$. The electron beam deviation is equal to:

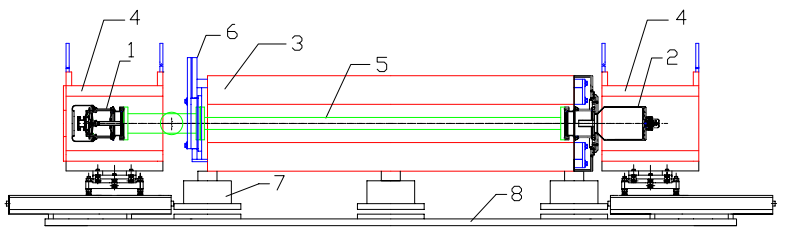

Figure 1: Prototype of the beam-beam compensation device. 1 - electron gun, $2-$ collector, $3-4 \mathrm{kG}$ solenoid with 16 pairs of dipole corrector coils, 4- gun and collector solenoids, 5-vacuum pipe, 6-current input/outputfor main solenoid, 7-adjustable supports, 8- assembly table.

$$
x_{e}(z)=\int^{z}\left(B_{\perp} / B_{\|}\right) d z^{\prime} .
$$

The requirement on the transverse field then depends on the wavelength $\lambda$ of the field imperfection:

$$
B_{\perp}(\lambda) / B_{\|} \lesssim \Delta x_{e} 2 \pi / \lambda .
$$

For the longest relevant wavelength $\left(\lambda \approx 2 L_{e}\right)$ we get $B_{\perp} / B_{\|} \lesssim 1.5 \cdot 10^{-4}$ that is comparable with what is typically required in electron cooling devices. However, in our case the requirement becomes less stringent for shorter wavelength $\lambda$, in contrast with electron cooling magnetic systems. The problem of the field straightness measurement and its correction is nevertheless an important issue, which inspired development of a suitable technique that has been tested at a prototype of the beam-beam compensation device recently commissioned at Fermilab [2].

The goals of the prototype (see Figure 1) were to obtain a low energy ( $\lesssim 10 \mathrm{kV}$ ) 2-meter long electron beam with total current up to $2 \mathrm{~A}$ propagating in a precise solenoid magnet, to test the current modulation in a few MHz bandwidth, and to study the electron beam dynamics. Parameters of the experimental installation are about the same as for the full scale device to be used in the Tevatron, except for a lower magnetic field and thus lower electron current density in the central solenoid.

The "electron-lens" prototype magnetic system consists of the main solenoid ( 2 meter long, $4 \mathrm{kG}$ maximum field) and two additional solenoids ( 0.5 meter long, maximum field about $4 \mathrm{kG}$ ) where the gun and collector are installed. The solenoids of the prototype are warm while the full scale device will require the main solenoid to be superconductive. The field in all solenoids can be changed independently, which provides a possibility to compress adiabatically the electron beam in the main solenoid and also to optimize the collector efficiency (see Figure 2). The field straightness tolerance is applicable only to the field in the main solenoid. 
The main solenoid consists of two 0.96 meter long sections. The sections have been designed and built for an electron cooling set-up MOSOL [3]. The sections are placed on adjustable supports which provides their relative alignment. The coil of the main solenoid is a precisely fabricated single layer copper spiral with 32 turns per section and with internal diameter $20 \mathrm{~cm}$. The spiral cross-section is $26 \times 34 \mathrm{~mm}$, the current corresponded to $4 \mathrm{kG}$ field is about $10 \mathrm{kA}$. In order to provide reliable electrical connection between two sections with such a high current density, precisely matched flat surfaces of the corresponding spiral edges of two sections were connected with high stress provided by four titanium wedge bolts. Total force applied to the contact is about several tons. A thin indium foil is placed between surfaces to increase the effective area of the contact. The current is returned to the commutation end by 8 straight copper bars which have been aligned in parallel with the solenoid axis. Special measures have been taken to distribute the return current evenly amongst the bars in order to ensure that the field direction coincide with the geometrical axis of the main solenoid.

Fine alignment of the magnetic field is provided by 4 pairs of $50 \mathrm{~cm}$ long and 12 pairs of $12 \mathrm{~cm}$ long dipole corrector coils installed atop the main solenoid in both planes. The short coils are placed at the ends of sections and the long coils in the center. Each pair of coils is fed by separate $\pm 2 \mathrm{~A}$ power supply and can produce up to $\pm 20 \mathrm{G}$ at the solenoid axis. One additional dipole coil is installed in the gap between collector solenoid and the main solenoid. This coil compensates field distortion produced by current commutation of the main solenoid.

\section{FIELD MEASUREMENTS AND ALIGNMENT}

The probe that allows to measure the direction of magnetic field is a flat mirror with attached magnetic arrow (see Figure 3). The mirror has 2 degrees of freedom so that it is oriented along magnetic field lines allowing optical measurements of the field imperfection. The diameter of the reflective surface is $21 \mathrm{~mm}$, diameter and length of the arrow are

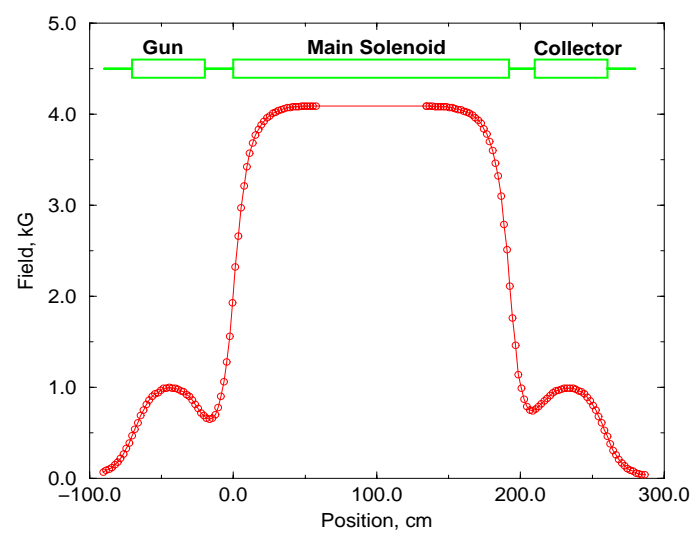

Figure 2: Longitudinal magnetic field in the "electron lens" prototype.


Figure 3: Mirror with magnetic arrow. 1- arrow, 2- reflective surface.

$2 r_{a}=5 \mathrm{~mm}$ and $l_{a}=25 \mathrm{~mm}$. The mirror is installed on a special nonmagnetic wheeled cart. The mirror with the magnetic arrow is the most delicate part of this measurement scheme. The mirror has to be sufficiently flat, it must be well balanced and the friction in axis should be small. We use a mirror previously used for similar measurements at the IUCF electron cooler [4].

Autocollimators are widely used for similar measurements in electron cooling devices. Since our field quality requirements are less stringent, we have chosen less expensive technique based on a use of a laser beam and twodimensional Position Sensitive Device (PSD). Signal of a PSD is proportional to the laser spot displacement with respect to the PSD center.

The scheme of measurements is shown in the Figure 4. Diode laser delivers a red light beam with $\lambda=670 \mathrm{~nm}$, initial beam size $3 \mathrm{~mm}$ and total beam divergence $0.35 \mathrm{mrad}$, which provides acceptably small beam spot at the mirror. The PSD (produced by Hamamatsu) has a $13 \times 13 \mathrm{~mm}^{2}$ working area, sensitivity of about $1 \mathrm{~V} / \mathrm{mm}$, and resolution of about $5 \mu \mathrm{m}$. An optical filter in front of the PSD is necessary to separate only the laser wavelength in order to decrease background of the PSD. The laser, a beam splitter, the filter and the PSD were assembled all together and placed at about $1 \mathrm{~m}$ from the collector solenoid. The assembly was placed on a mover which allowed position and angular correction in two transverse planes in order to align the system with respect to the axis of the main solenoid.

An aluminum tube (380 $\mathrm{cm}$ long, $10 \mathrm{~cm}$ diameter) was placed inside the solenoid on axis to provide a guide for the mirror cart. The cart can move along the tube by means of a plastic belt and a stepping motor placed in the field free region near one end of the tube. Standard Hall probes with longitudinal and transverse sensors can be optionally installed on the cart. The PSD signals are preamplified and

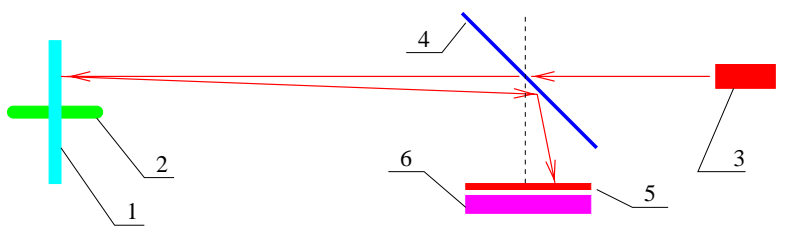

Figure 4: Scheme of measurements. 1 and 2 - mirror with magnetic arrow, 3- diode laser, 4- beam splitter, 5- optical filter, 6- PSD. 


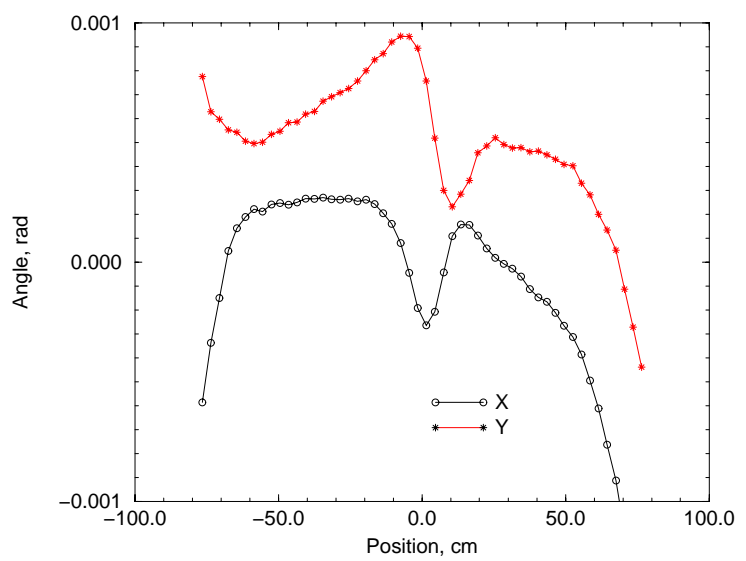

Figure 5: Angular deviation of the main solenoid field. Zero position corresponds to the middle of the main solenoid.

measured by an ADC. The entire system is controlled by LabView (TM) based data acquisition system.

Precision of the method depends on several factors. If the magnetic field is weak, then the precision is determined by friction in the mirror axises. The measured angular precision (repeatability) of the mirror is $\alpha_{\mathrm{rms}} \approx 1.5 \times$ $10^{-4}(1 \mathrm{kG} / B)^{2}$. The dependence $\alpha_{\mathrm{rms}} \propto 1 / B^{2}$ is valid for $B \lesssim 1.5 \mathrm{kG}$, then the arrow is saturated and the dependence becomes $\alpha_{\mathrm{rms}} \propto 1 / B$. The precision of measurements in a higher field, at about $4 \mathrm{kG}$, is also determined by other factors such as vibrations, electrical noises, etc., and was of the order of 10-20 $\mu \mathrm{rad}$.

The region of linearity of the system is determined by the size of the PSD working area and by the laser spot size at the PSD. The linearity range in terms of the spot displacement on the PSD face is found to be about $\pm 6 \mathrm{~mm}$, that corresponds to $\pm 1 \mathrm{mrad}$ for the mirror located $3 \mathrm{~m}$ away from the PSD. The field deviation at the ends of the main solenoid is larger than this value. However, this limitation is not significant for the procedure of the field alignment by dipole corrector coils, since the field deviation will decrease in the

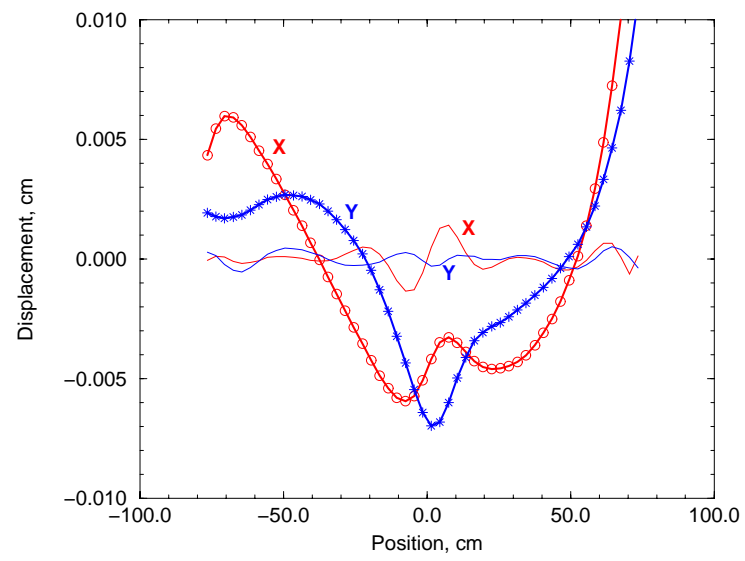

Figure 6: Electron beam displacement without (stars and circles) and with correction by dipole coils (simulation). iterative procedure of alignment.

Angular deviation of the magnetic field $B_{\perp} / B_{\|}$in the main solenoid is shown in Figure 5. One can see that the initial field quality is mostly determined by the edge effects. Exposure to the support table on which the solenoid is installed and current commutators at the ends of the main solenoid are thought to be major disturbances. Deviations of the field in the middle are caused by the force that provides electrical contact between sections and which also deforms the spiral of the solenoid at the place of connection, resulting in the field perturbation.

Electron beam trajectory in the measured magnetic field can be calculated using Eq.1 (see Figure 6). The rms trajectory displacement over $80 \%$ of the length of the main solenoid was found to be $\sigma_{x}=0.05 \mathrm{~mm}$ and $\sigma_{y}=$ $0.04 \mathrm{~mm}$, which satisfies the requirements for the "electron lens". Use of corrector coils allows further field quality improvement. The beam trajectory corresponded to simulated alignment of the magnetic field by use of dipole coils is shown in the Figure 6. The rms orbit displacement in this case is $\sigma_{x}=0.005 \mathrm{~mm}$ and $\sigma_{y}=0.003 \mathrm{~mm}$ that is 10 times less than required. These results suggest that the same field quality in the full scale beam-beam compensation device can be achieved with few dipole correctors at the ends of the main $5 \mathrm{~T}$ solenoid.

\section{CONCLUSION}

The guiding solenoidal magnetic field of the prototype of the beam-beam compensation device has been measured using a simple optical method based on a use of a mirror with a magnetic arrow, a diode laser and a position sensitive device. The field quality in the test facility was found to be good enough in the central part of the main solenoid while a modest correction by dipole coils is required at the ends of the solenoid. The results will help to determine a proper field alignment technique for the full scale beambeam compensation device.

\section{ACKNOWLEDGEMENT}

We acknowledge useful discussions with S. Nagaitsev and A. Shemiakin and valuable technical assistance came from L. Arapov, A. Chupira, G. Kuznetsov, J. Santucci and A. Warner.

\section{REFERENCES}

[1] V.Shiltsev, D.Finley, A.Sery, V.Danilov, "Compensation of Beam-Beam Effects in the Tevatron Collider with Use of Electron Beams", these Proceedings; see also FNAL-Pub98/260 (1998).

[2] C.Crawford, et. al, "Prototype "Electron Lens" Set-Up for the Tevatron Beam-Beam Compensation", these Proceedings.

[3] L. Arapov, et. al, "Precision Solenoid for Electron Cooling", in Proc. Int. Conf. Charged Particle Accelerators, Novosibirsk, vol.1, (1986), p.341.

[4] The mirror was kindly provided to us by S. Nagaitsev. 